Background Culture is insensitive for detecting pharyngeal gonorrhoea but is critical for determining antimicrobial resistance. Sampling technique appears to be important for optimal isolation of pharyngeal gonorrhoea, however, there are no published studies on the specific anatomical areas within the pharynx that should be targeted. The aim of this study was to compare isolation rates of gonorrhoea from the tonsils and posterior oropharynx.

Method Men who had sex with men attending the Melbourne Sexual Health Centre who screened positive for pharyngeal gonorrhoea using modified Thayer Martin medium were recalled and reswabbed prior to treatment. The repeat swabs consisted of careful swabbing from both tonsils followed by swabbing of the posterior oropharynx using a separate swab. These were plated onto separate media and cultured.

Results To date $61 \mathrm{MSM}$ who screened positive for pharyngeal gonorrhoea have been recalled for repeat swabbing. The median interval between the initial positive screening test and repeat swabbing was 7 days. The positivity rates from the repeat swabs of the tonsils and posterior oropharynx were 69\% $(n=41)$ and $52 \%$ $(\mathrm{n}=32)$ respectively. The repeat tonsillar and posterior oropharyngeal swabs were both positive in $51 \%(n=31)$ of men and were both negative in $30 \%(n=18)$ of men. Eleven men $(18 \%)$ had positive tonsillar and negative oropharyngeal results while one man had positive oropharyngeal and negative tonsillar results $(p<0.01)$. The results of concurrent nucleic acid amplification testing will be presented.

Conclusion These interim study results indicate that Neisseria gonorrhoeae can be cultured from the tonsils as well as the posterior oropharynx. While the positivity rate from tonsillar swabbing was higher than swabbing of the posterior oropharynx, sampling from only one of these sites had poor sensitivity. Many cases of pharyngeal gonorrhoea appear to be transient and self limiting.

\section{P2.034 SAMPLING TECHNIQUE IS IMPORTANT FOR OPTIMAL ISOLATION OF PHARYNGEAL GONORRHOEA}

doi:10.1136/sextrans-2013-051184.0299

'M Mitchell, 'V Rane, 1,2 C K Fairley, 3,4D M Whiley, 1,2.5 C S Bradshaw, 1,2M Bissessor, ${ }_{1,2}$ M Y Chen. 'Melbourne Sexual Health Centre, Alfred Health, Melbourne, Australia, ${ }^{2}$ Melbourne School of Population and Global Health, University of Melbourne, Melbourne, Australia; ${ }^{3} T$ The University of Queensland, St Lucia, St Lucia, Australia; ${ }^{4}$ Queensland Paediatric Infectious Diseases Laboratory, Royal Children's Hospital, Brisbane Herston, Australia; ${ }^{5}$ Department of Epidemiology and Preventive Medicine, Monash University, Melbourne, Australia

Background Culture is insensitive for the detection of pharyngeal gonorrhoea but isolation is pivotal to antimicrobial resistance surveillance. The aim of this study was to ascertain whether recommendations provided to clinicians on pharyngeal swabbing technique could improve gonorrhoea detection rates and to determine which aspects of swabbing technique are important for optimal isolation.

Methods This study was undertaken at the Melbourne Sexual Health Centre, Australia. Detection rates for pharyngeal gonorrhoea among clinicians were compared before (June 2006-May 2009) and after (June 2009 - June 2012) recommendations on swabbing technique were provided. Associations between detection rates and reported swabbing technique obtained via a clinician questionnaire were examined.

Results The overall yield from testing before and after provision of the recommendations among 28 clinicians was 1.6\% (134/8586) and $1.8 \%(264 / 15,046)$ respectively $(p=0.17)$. Significantly higher detection rates were seen following the recommendations among clinicians who reported: a change in their swabbing technique in response to the recommendations $(2.1 \%$ versus $1.5 \% ; p=0.004)$; swabbing a larger surface area $(2.0 \%$ versus $1.5 \%$; $p=0.02)$; applying more swab pressure $(2.5 \%$ versus $1.5 \%$; $<0.001)$; and a change in the anatomical sites they swabbed $(2.2 \%$ versus $1.5 \%$; $p=0.002)$. The predominant change in sites swabbed was an increase in swabbing of the oropharynx - from a median of $0 \%$ to $80 \%$ of the time.

Conclusion More thorough swabbing improves isolation of pharyngeal gonorrhoea using culture. Clinicians should receive training to ensure swabbing is performed with sufficient pressure and that it covers an adequate area that includes the oropharynx.

\section{P2.035 AS NUCLEIC ACID AMPLIFICATION TESTING FOR NEISSERIA GONORRHOEAE INCREASES IS CULTURE CONFIRMATION DECREASING?}

doi:10.1136/sextrans-2013-051184.0300

M E 0 Perry, G Haidari, J A White. Guys and St Thomas' NHS Foundation Trust, London, UK

Background With the advent of nucleic acid amplification tests (NAATs) to detect Neisseria gonorrhoeae (GC) the use of selective culture is now reserved largely for symptomatic patients or confirmation of GC NAAT-positive results prior to treatment. The importance of culture remains greater than ever for monitoring antimicrobial susceptibility in the face of emerging GC resistance.

Aim We hypothesise that there is decreased likelihood of culture sampling at initial testing prior to treatment and thus there might be fewer culture samples available for antimicrobial susceptibility.

Methods A list of GC-positive cases in 2011 and 2012 was generated from the laboratory. All cases were reviewed and recorded as having had: culture not taken, culture taken \& negative, culture taken \& positive.

Annual cases were compared for the proportion being cultured prior to treatment, culture positivity rate and changes over time were assessed.

\section{Results}

Conclusion In our busy urban Genito Urinary Medicine (GUM) clinic we have shown that despite the increased ease and sensitivity of GC NAATs, GC culture has been sustained. The increase in the number of GC cases seen in 2012 is commensurate with an increase in testing episodes, yet the proportion with cultures taken pre-treatment actually increased from $64 \%$ in 2011 to $78 \%$ in 2012. Nonetheless, culture positivity has remained stable at $50 \%$ between 2011 and 2012. This is likely a result of our large MSM cohort, with high rates of rectal \& throat infections in

Abstract P2.035 Table 1 Numbers of GC NAAT-diagnosed infections and associated culture results

\begin{tabular}{lllllll}
\hline & 2011 & \multicolumn{5}{l}{$\mathbf{2 0 1 2}$} \\
\cline { 2 - 6 } & GC NAAT positive & GC culture taken & GC cultures positive & GC NAAT positive & GC culture taken & GC cultures positive \\
\hline Total & 1056 & $675(64 \%)$ & $336(50 \%)$ & 1325 & $1030(78 \%)$ & $518(50 \%)$ \\
Rectum & 408 & $235(58 \%)$ & $116(49 \%)$ & 509 & $386(76 \%)$ & $221(57 \%)$ \\
Throat & 424 & $239(56 \%)$ & $35(15 \%)$ & 556 & $400(72 \%)$ & $70(18 \%)$ \\
Urine/urethral & 222 & $201(91 \%)$ & $185(92 \%)$ & 260 & $244(94 \%)$ & $227(93 \%)$ \\
\hline
\end{tabular}

